\title{
Nuevos registros de insectos fitófagos asociados a canelo (Drimys winteri Forst.) en La Araucanía
}

\author{
New registers of phytophagous insects associated with canelo in La Araucania \\ Priscilla Brebi M. ${ }^{1,2}$, Alfonso Aguilera ${ }^{1}$, Nelson Ojeda $^{1}$, Ramón Rebolledo ${ }^{1}$
}

\section{RESUMEN}

En este trabajo se planteó determinar las especies de insectos asociados a Drimys winteri Forst. (canelo) en dos lugares de La Araucanía. Uno correspondiente a un sector rural (Sector Villa Comuy) y otro urbano (Comuna de Temuco). En cada sector se eligieron tres árboles para recolectar las muestras. El diseño experimental utilizado en la presente investigación correspondió a parcelas completamente al azar, eligiendo tres unidades muestrales con un total de ocho muestreos en la temporada 2006. Los resultados de los muestreos permitieron determinar como nuevos registros para La Araucanía las siguientes especies: Trialeurodes unadutus (Hemiptera, Aleyrodidae); Heliothrips haemorrhoidalis (Thysanoptera, Thripidae) y Hemiberlesia rapax (Hemiptera, Diaspididae). Además, se observó un lepidóptero enrollador de hojas, pero no fue posible su determinación dado que solamente se registró el daño causado en la hoja. En ambas áreas se encontraron las mismas especies pero con diferencias en los promedios y la abundancia relativa.

Palabras clave: Insectos, fitófagos, siempreverde, equidad.

\section{ABSTRACT}

In this work it was necessary to determine the species of insects associated with Drimys winteri Forst. (canelo) at two locations of La Araucania. One corresponds to a rural sector (Sector Comuy), and another city (Temuco). In each sector were elected three trees to collect samples. The experimental design used in this research plots corresponded to completely at random, choosing three units show with a total of eight different dates in the 2006 season. The results of the sampling helped identify as new records for La Araucania the following species: Trialeurodes unadutus (Hemiptera, Aleyrodidae); Heliothrips haemorrhoidalis (Thysanoptera, Thripidae) and Hemiberlesia rapax (Hemiptera, Diaspididae). In addition, there was a winder lepidopterous leaves, but his determination was not possible because there was only damage caused in the leaf. Both areas were the same species but with differences in the averages and the relative abundance.

Key words: Insects, phytophagous, equity.

\section{Introducción}

El principal tipo forestal que registra el catastro vegetacional de Chile corresponde al tipo siempreverde, con una superficie de 4,35 millones de hectáreas, las que representan un 30,9\% del total del bosque nativo de Chile. Dentro de éstas se encuentran los renovales de canelo ocupando una superficie de 266.303 ha (INFOR-CONAF, 1997).

En 1990 el cien por ciento de la producción maderera de canelo se generó en la Región de Los Lagos, tendencia que se mantuvo en 1993 (INFORCORFO, 1995). Según la misma fuente, la producción de madera aserrada de canelo representó en el 0,2\% del total nacional de todas las especies en el año 1990 y el 0,3\% para 1993. Según Corvalán (1987), el canelo destaca también por su aptitud papelera, ya que posee un largo de fibra mayor al Pinus radiata y a la vez tiene aptitud favorable para la fabricación de tableros de partículas.

Otro de los usos de canelo es medicinal, ya que sus hojas son utilizadas para aliviar dolores en general (Hoffmann, 1982). Además, la corteza posee propiedades tónicas, usada como antiescorbútico y al mismo tiempo sirve para el reumatismo (Linetzky, 1940). Hoffmann (1982) señala que el canelo también

\footnotetext{
1,2 Doctorado en Ciencias mención Biología Celular y Molecular, Universidad de La Frontera. Casilla 45-D. Temuco, Chile. 045-393118. E-mail: pbrebi@ufro.cl

1 Facultad de Ciencias Agropecuarias y Forestales Universidad de La Frontera. Casilla 54-D. Temuco, Chile.
} 
se emplea como planta ornamental en parques y jardines. Peña (1995) trabajó el uso de canelo como insecticida, ya que este posee componentes químicos como el poligodial, sustancia que al ser ingerida por los insectos les inhibe la capacidad de alimentarse de las hojas o de otra parte del vegetal.

En Chile el canelo se distribuye desde el río Limarí ( $30^{\circ}$ latitud Sur) hasta el Cabo de Hornos (56 latitud sur). Sin embargo, su máxima expresión la alcanza en los bosques laurifolios (Rodríguez et al., 1983). Esta especie se concentra mayoritariamente en la Región de Los Lagos y alcanza su mejor desarrollo en la zona de Chiloé (Donoso y Cabello, 1978; Donoso, 1981). El canelo adquiere mayor importancia en el tipo forestal siempreverde, donde pasa a ser la especie principal en los subtipos ñadis y renovales de canelo (Donoso, 1981).

Cabe destacar que existen escasos trabajos de insectos presentes en canelo. Sin embargo, Fernández (1985) describe que los principales insectos presentes en canelo son larvas de lepidópteros de la familia Tortricidae que es un pegador de hojas y un díptero de la familia Cecidomyiidae que produce agallas. Se ha detectado un cortador de tallo, pero aún no ha sido determinado. Peña (1996) agrega que además de Lepidoptera, algunos Hymenoptera también atacan al canelo.

En cuanto a daños en la regeneración de canelo, Loewe (1987) encontró daños provocados por larvas de lepidópteros denominadas comúnmente como "chubas" sin entregar el nombre científico. Estas dejan los tallos de las plántulas desprovistos de hojas (INFOR-CONAF, 1997).

Klein \& Waterhouse (2000) se refieren a insectos causantes de daños en canelo nominando a Aegorhinus nodipennis (Hope) (Coleoptera, Curculionidae), Ythutomus farmosus (Butler) (Lepidoptera, Yponomeutidae), Ythutomus valdivianus (Beeche y Parra) (Lepidoptera, Yponomeutidae).

Como se puede apreciar, existe una escasez de trabajos sobre insectos asociados a canelo y, por ello, se ha planteado como objetivo en este trabajo realizar una prospección de insectos fitófagos en hojas y fustes de Drimys winteri, y también estimar su abundancia relativa en dos sectores de La Araucanía.

\section{Materiales y Métodos}

Para determinar los insectos presentes en canelo se efectuaron prospecciones en dos lugares de La Araucanía, uno en la zona urbana y el otro en la zona rural. La zona rural correspondió a la localidad de Comuy, comuna de Pitrufquén, en el km 31 camino Pitrufquén a Toltén, más específicamente en el Huso 18 H 0676419 UTM 5672950, donde existía un bosque de canelo asociado fundamentalmente a roble (Nothofagus obliqua (Mirb.) Oerst.) y coigüe (Nothofagus dombeyi (Mirb.) Oerst.). La zona urbana correspondió a la comuna de Temuco, Campus Andrés Bello de la Universidad de La Frontera, Huso 18 H 0709285 UTM 57709413, en la cual canelo forma parte del entorno como árbol ornamental.

Para la obtención de datos se muestrearon hojas cada 15 días, completando un total de ocho muestreos por área, estos fueron realizados entre los meses de enero a abril del 2006. La hora de muestreo fue desde las 11:00 AM hasta las 4:00 $\mathrm{PM}$, en ambas situaciones.

Para ambos sectores la colecta se realizó en forma manual, eligiendo para ello tres árboles con características similares. En cada árbol se eligieron dos alturas: alta $\geq 1,30 \mathrm{~m}$ y baja $<1,30 \mathrm{~m}$; dos estratos (exterior e interior), y cuatro orientaciones (norte, sur, este y oeste), colectando de cada uno de los puntos tres hojas en forma aleatoria, completando de esta manera un total de 48 hojas por árbol y 144 hojas por muestreo.

Las hojas fueron almacenadas en bolsas de plástico previamente rotuladas y transportadas al Laboratorio de Entomología de la Facultad de Ciencias Agropecuarias y Forestales de la Universidad de La Frontera. Una vez en el laboratorio las muestras se mantuvieron $\mathrm{a} \pm 2{ }^{\circ} \mathrm{C}$ en un refrigerador para su posterior revisión e identificación de los insectos.

Para estimar la abundancia relativa de los insectos asociados a canelo se utilizó el diseño experimental aleatorio simple, eligiendo tres unidades muéstrales completamente al azar, con ocho repeticiones cada una, completando un total de ocho muestreos en cada área de estudio.

El análisis estadístico fue realizado a través del software SPSS versión 11,0 en español, contrastando las variables de insectos encontrados en el área rural y urbana a través de la prueba no paramétrica de Kruskal-Wallis (Visauta, 1997). También se utilizó estadística descriptiva para medidas de tendencia se aplicaron algunos parámetros poblacionales en donde se evaluó la abundancia total, riqueza específica, abundancia relativa y la diversidad, esta última a través del índice de Shannon-Wiener (Krebs, 1985 y Moreno, 2001). 


\section{Presentación y Discusión de Resultados}

En el área rural y urbana se encontraron insectos causando daños en las hojas (Cuadro 1). Los insectos defoliadores y enrolladores mencionados por la literatura no se encontraron en los periodos de muestreo realizados en este trabajo.

Cuadro 1. Especies de insectos identificadas en el área urbana y rural (Temporada 2006).

\begin{tabular}{lccc}
\hline Especie & $\begin{array}{c}\text { Nombre } \\
\text { común }\end{array}$ & Orden & Familia \\
\hline $\begin{array}{l}\text { Heliothrips } \\
\text { haemorrhoidalis } \\
\text { (Bouché) }\end{array}$ & Trips. & Thysanoptera. & Thripidae \\
$\begin{array}{l}\text { Hemiberlesia rapax } \\
\text { (Comst.) }\end{array}$ & $\begin{array}{c}\text { Escama } \\
\text { rapaz }\end{array}$ & Hemiptera & Diaspididae \\
$\begin{array}{l}\text { Trialeurodes } \\
\text { unadutus Bak. \& } \\
\text { Moles }\end{array}$ & $\begin{array}{c}\text { Mosquita } \\
\text { blanca. }\end{array}$ & Hemiptera & Aleyrodidae \\
\hline
\end{tabular}

Los resultados difieren de Klein \& Waterhouse (2000), quienes señalan que los principales insectos asociados a canelo son Aegorhinus nodipennis (Hope), Ythutomus farmosus (Butler), Ythutomus valdivianus (Beeche y Parra), los cuales no fueron recolectados en este estudio. Esto pudo deberse a la época de muestreo del presente estudio (verano 2006).

El insecto encontrado en mayor porcentaje correspondió a Trialeurodes unadutus (75\%). En segundo lugar, Heliothrips haemorrhoidalis (12\%) y tercero Hemiberlesia rapax (13\%).

Respecto a Trialeurodes unadutus se puede observar en la figura 1 que existió en el área rural mayor población de individuos que en el muestreo realizado el 11 de marzo, superando a la mayor población del área urbana registrada el 20 de enero.

Hemiberlesia rapax se presentó en casi todos los muestreos, exceptuando el muestreo del 1 de abril, en el cual no se presentó ningún individuo, a diferencia del muestreo del 11 de marzo, el cual concentró el valor máximo. El rango de individuos encontrados fluctuó entre 0 y 32. El área rural denotó muy poco daño provocado por Heliothrips haemorrhoidalis; sin embargo, está presente en todos los muestreos realizados: el número de hojas dañadas fluctuó entre 7 y 20 , no presentando en ninguna de las hojas un daño cercano al cien por ciento, sino más bien afectó un pequeño porcentaje de la superficie foliar.
En el área urbana (Cuadro 1; Figuras 1a y 1b) se encontraron los mismos insectos anteriormente descritos para el área rural, sólo con una diferencia en las densidades de ellos, además la mayoría de las hojas presentaron daños significativos, radicando en la presencia de muy pocas hojas sanas, ambas situaciones evaluadas en relación al total de hojas muestreadas para dicho lugar. Aquí se encontró un mayor número de individuos de Hemiberlesia rapax (28\%), pero con una mayor incidencia en cuanto al daño de Heliothrips haemorrhoidalis (28\%), el mínimo de abundancia fue de Trialeurodes unadutus con apenas un $2 \%$.

Lo primero que se puede destacar para Hemiberlesia rapax (Comst.) es el aumento del

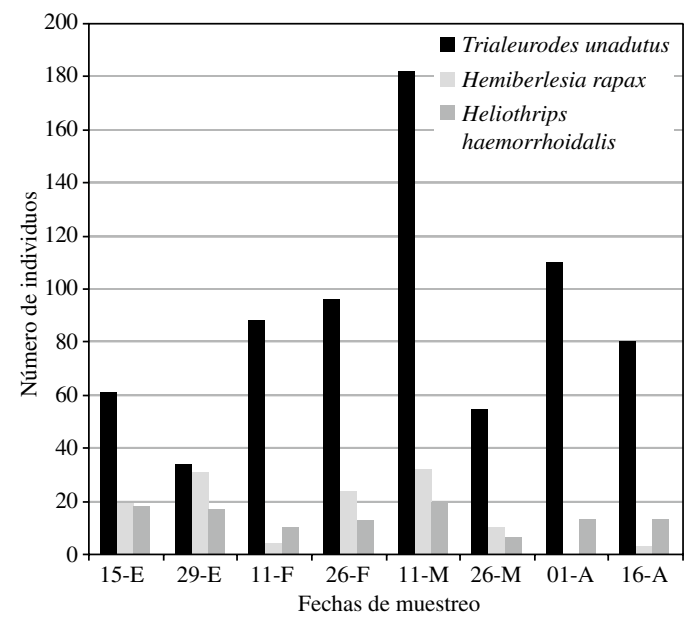

Figura 1a. Número de individuos por muestreo en el área rural (temporada 2006).

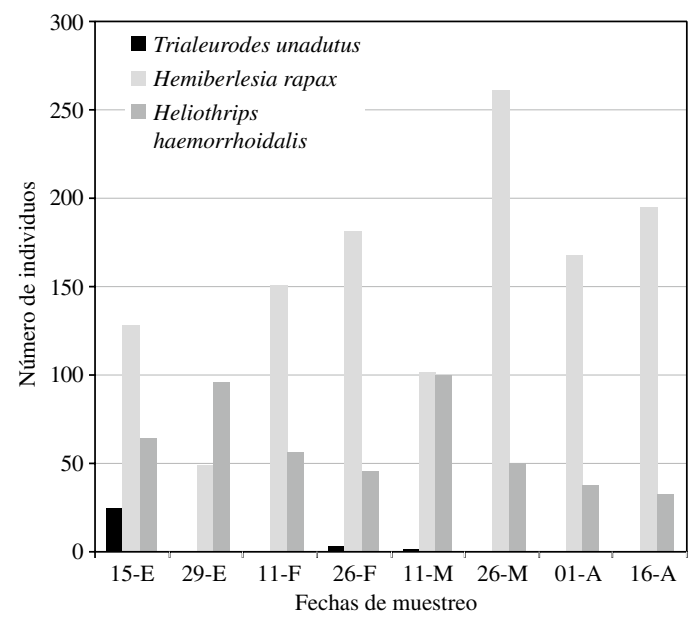

Figura 1b. Número de individuos por muestreo en el área urbana (temporada 2006). 
número de individuos encontrados, en relación al área rural, logrando un rango de aproximadamente 0 y 300 individuos. En el muestreo del 31 de marzo se obtuvo el máximo número de individuos de la especie, con una representación cercana a los 261 insectos. El mínimo se determinó en el muestreo del 3 de febrero, apenas logrando llegar a los 49 ejemplares, valor más alto que el máximo encontrado en el área rural.

En el caso de Heliothrips haemorrhoidalis (Figura 1a), se detectó daño en todos los muestreos, con mayor número de hojas afectadas en relación al área rural, fluctuando aproximadamente entre 33 y 99 hojas. A diferencia del área rural, las hojas presentan un daño cercano al cien por ciento de la superficie foliar. El muestreo del 17 de marzo concentró el mayor daño, a diferencia del muestreo del 24 de abril, el cual determinó un mínimo de hojas con daño provocado por dicha especie.

La abundancia relativa de Trialeurodes unadutus en el área rural (Figura 1a) fue de 34 ejemplares como mínimo con un máximo de 182 individuos. En el área urbana (Figura 1b) hubo muestras sin registro de Trialeurodes unadutus cuyo máximo fue de 25 individuos. La varianza de los datos para el área rural fue de 2.025,929, lo que indica datos muy variables y heterogéneos, mientras para el área urbana correspondió a 75,071, representando una menor variabilidad, y a la vez implica que los datos obtenidos fueron más homogéneos (Figura $2 \mathrm{~A}$ ). El promedio poblacional fue de 88,25 ejemplares para el área rural y 3,75 para el área urbana, lo que denota una diferencia significativa existente entre ambas áreas.

Hemiberlesia rapax presentó en ambas áreas diferencias en cuanto a la variabilidad, con una varianza de 165,12 en el área rural y 4066,83 en la urbana, indicando datos más variables donde canelo crece en forma ornamental con un valor muy significativo (Figura $2 \mathrm{~B}$ ).

Además; la media de ambas áreas es muy distinta, por lo que se puede percibir que existe diferencia significativa de las poblaciones de Hemiberlesia rapax $(\mathrm{P}<0,05)$ entre los dos lugares de estudio de la Región de La Araucanía.

En la figura $2 \mathrm{C}$ se observa que el área urbana está más dañada que la rural, debido a que presenta mayor cantidad de hojas afectadas por Heliothrips haemorrhoidalis. El área urbana presentó una mayor variabilidad de los datos; esto se determinó al observar la varianza, la cual es de 625,554,
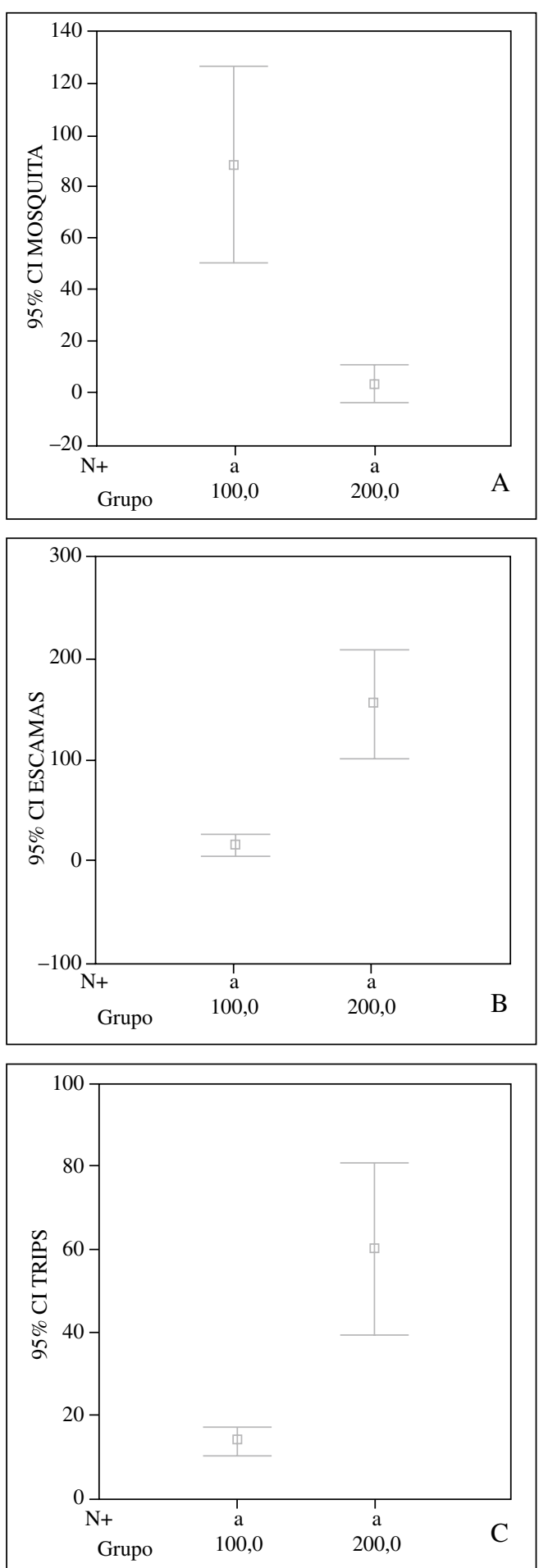

Figura 2. Comparación de medias e intervalo de confianza entre las dos áreas de estudio (temporada 2006). 1. Área rural. 2. Área urbana. A: Trialeurodes unadutus; B: Hemiberlesia rapax; C: Heliothrips haemorrhoidalis. 
lo que indica datos más heterogéneos que en el área rural.

Todo esto lleva a pensar que existe una significativa diferencia entre las poblaciones de Heliothrips haemorrhoidalis de las dos áreas de estudio de la Región de La Araucanía $(\mathrm{P}<0,05)$.

Cuadro 2. Abundancia total (N), riqueza específica (S), abundancia relativa $\mathrm{AB}(\%)$, diversidad ( $\left.\mathrm{H}^{\prime}\right)$, diversidad máxima (H'máx.) e índice de equidad (J) de los insectos encontrados en canelo en dos sectores de estudio (temporada 2006)

\begin{tabular}{lcc}
\hline & \multicolumn{1}{c}{ Rural } & Urbana \\
\hline Abundancia total (N) & 940 & 1746 \\
Riqueza específica (S) & 3 & 3 \\
AB(\%) entre las dos áreas & $35 \%$ & $65 \%$ \\
Shannon-Wiener (H') & 1,05805136 & 0,966470135 \\
H' máx. & 1,5849625 & 1,584962501 \\
Equidad (J) & 0,66755608 & 0,609774764 \\
\hline
\end{tabular}

Los parámetros poblacionales aplicados indicaron que la abundancia total registrada representó un mayor número de ejemplares en el área urbana, indicando aquí mayores problemas sanitarios en comparación con el área rural. En tanto la riqueza específica en ambas áreas se representó por tres especies, lo que implica que ambos lugares son similares en relación a dicha variable. La mayor abundancia relativa $(\mathrm{AB} \%)$ con respecto a las dos áreas se situó en la zona urbana, la cual presentó un $65 \%$ del total de individuos encontrados, haciéndola muy representativa de los insectos presentes en canelo, ya que en esta área se registró el mayor número de individuos de todo el estudio.

En el área rural la equidad fue de un 0, 66, lo que indica que un $66 \%$ de los individuos están repartidos dentro de las distintas especies presentes en dicha área. En el área urbana la equidad es de un 0,60 lo que indica que un $60 \%$, de los individuos están repartidos dentro de las distintas especies presentes en dicha área, este valor es un poco más bajo que el obtenido en el área rural. Cabe destacar que no existe una clara diferencia entre la equidad del área urbana y rural, ya que los valores determinados no son significativamente diferentes entre ambas áreas de estudio.

\section{Conclusiones}

Del presente estudio se concluye que:

- Heliothrips haemorrhoidalis (Bouche), Hemiberlesia rapax (Comst.) y Trialeurodes unadutus Bak. \& Moles corresponden a nuevos registros de insectos asociados a canelo en $\mathrm{La}$ Araucanía.

- En ambas áreas se encontraron los mismos insectos, pero con una diferencia en la abundancia e incidencia de éstos, ya que en el área urbana el porcentaje de daño fue mayor que en la rural. Esto fue determinado mediante la abundancia relativa, la cual arrojó un mayor valor en la ciudad.

- Canelo presentó daños provocados por defoliadores no determinados tanto internos como externos, siendo éstos más perjudiciales en el área urbana.

- Trialeurodes unadutus presentó una mayor abundancia relativa en el área rural. A diferencia de esto, fue la especie Hemiberlesia rapax la que presentó una mayor abundancia en el área urbana, pero realizando un mayor daño allí la especie Heliothrips haemorrhoidalis.

- Trialeurodes unadutus presentó una correlación estadísticamente significativa entre las áreas rural y urbana. Esta situación se repitió para las otras dos especies determinadas en el estudio.

- Las dos áreas de estudio arrojaron igual valor para la riqueza específica, representada solo por tres especies. La equidad es mayor en el área rural (66\%), sin embargo en comparación con el área urbana $(60 \%)$ no es un valor muy significativo. 


\section{Literatura Citada}

Corvalán, P.

1987 “El canelo: ¿Pino Radiata del Bosque Nativo?’. Revista Chile Forestal. pp. 22-24.

Donoso, C.

1981 Tipos forestales de los bosques nativos de Chile. Investigación y desarrollo forestal. Santiago, Chile. 70 p.

Donoso, C. y Cabello, A.

1978 Antecedentes fenológicos de especies leñosas chilenas. Ciencias forestales 2: 31-42.

Fernández, J.

1985 Propagación germinativa y vegetativa de Drimys winteri J.R, et G. Faerster. Tesis de Ingeniero Forestal, Facultad de Ciencias Forestales, Universidad Austral de Chile. Valdivia, Chile. 163 p.

Hoffmann, A.

1982 Flora silvestre de Chile, zona austral. Árboles, arbustos y enredaderas leñosas. Ediciones Fundación Claudio Gay. Santiago, Chile. 258 p.

INFOR-CONAF. Instituto Forestal-Corporación Nacional Forestal.

1997 Monografía de Drimys winteri. Santiago. Chile. 65 p.

INFOR-CORFO, Instituto Forestal-Corporación Nacional Forestal

1995 Antecedentes de mercado para canelo. Manuscrito INFOR-Valdivia por convenio INFOR-CONAF. $60 \mathrm{p}$.

Klein C. \& Waterhouse, D.F.

2000 Distribution and importance of arthropods associated with agriculture and forestry in Chile. (Distribución e importancia de los artrópodos asociados a la agricultura y silvicultura en Chile). ACIAR, Canberra, Australia. Monograph No 68. $231 \mathrm{p}$.
Krebs, Ch.

1985 Ecología: Estudio de la Distribución y la Abundancia. Segunda Edición. Instituto Ecológico de Recursos Animales. Universidad de Columbia Británica. D.F., México. 753 p.

Linetzky, A.

1940 Determinación del ácido ascórbico en la corteza de canelo (Drimys winteri Forst.). Tesis de Químico-Farmacéutico. Facultad de Biología y Ciencias Médicas. Universidad de Chile. Santiago. 95 p.

Loewe, V.

1987 Evaluación de la regeneración natural de canelo Drimys winteri Forst. en la X Región. Tesis Ingeniero Forestal, Facultad Ciencias Agrarias y Forestales. Universidad de Chile. Santiago, Chile. 205 p.

Moreno, C.

2001 Métodos para medir la biodiversidad. Centro de Investigaciones Biológicas. Universidad Autónoma del Estado de Hidalgo, México. Vol. 1. 83 p.

Peña, L.

1996 Introducción al estudio de los insectos de Chile. Editorial Universitaria. Santiago, Chile. 253 p.

Peña, C.

1995 Efecto de poligoidal extraído de corteza del canelo, Drimys winteri Forst., sobre algunos insectos de importancia agrícola. Tesis Ingeniero Agrónomo. Universidad de La Frontera. Temuco, Chile. $86 \mathrm{p}$.

Rodríguez, R.; Matthei, O. y Quezada, M. 1983 Flora arbórea de Chile. Ediciones de la Universidad de Concepción. Concepción. Chile.

Visauta, B.

1997 Análisis estadístico con SPSS para Windows. Estadística básica. Ed. McGraw-Hill/Interamericana de España. 332 p. 